\title{
AN AHP MODEL FOR BRINGING EXPERTS TO CONSENSUS ON MEDICAL PAYMENT STANDARDS *
}

\author{
Yuan-Huei HUANG ${ }^{1}$ Pei-Yeh CHANG ${ }^{2}$ \\ Chih-Young HUNG ${ }^{3}$ Kuei-Ing WANG ${ }^{4}$ King-Jen CHANG \\ ${ }^{1}$ Department of Surgery, Hsin-Chu General Hospital, Taiwan, China \\ pshyh@tpts4.seed.net.tw ( $\square)$ \\ ${ }^{2}$ Department of Pediatric Surgery, Chang Gung Children's Hospital, Chang Gung University, \\ College of Medicine, Taiwan, China, pyjchang@cgmh.org.tw \\ ${ }^{3}$ Institute of Management of Technology, National Chiao Tung University, Taiwan, China \\ cyhung@cc.nctu.edu.tw \\ ${ }^{4}$ Department of Information Management, Ming Hsin University of Science and Technology, Taiwan, China \\ kywang54@ms25.hinet.net \\ ${ }^{5}$ Department of Surgery, National Taiwan University Hospital, Taiwan, China \\ kingjen@ha.mc.ntu.edu.tw
}

\begin{abstract}
Many countries with health insurance systems conduct periodic payment standards reform. How to reach consensus in setting payment standards among different specialties with different agendas has become a critical issue. The purpose of this study is to construct an analytic hierarchy process (AHP) model to obtain judgments from experts about the importance of "factors related to establishing payment standards in the national health insurance program". Under this goal, the first tier contains four evaluation aspects, and the second tier contains sixteen evaluation criteria divided into four groups. The AHP model was then used to collect and combine the opinions of experts through an empirical study. The results can be directly used to formulate standard values as the basis for establishing payment standards. The results of our study strongly support that an AHP model is effective in forming a consensus among surgical specialists.
\end{abstract}

Keywords: National Health Insurance, Analytic Hierarchy Process, payment standards

\section{Introduction}

Many countries with health insurance systems conduct periodic payment standards adjustments. The main reasons are to address continually rising health care costs and to ensure an equitable allocation of resources. From the

\footnotetext{
* This study is deeply indebted to the Taiwan's Bureau of National Health Insurance, Department of Health, Executive Yuan for providing research funding (BNHI Year 2002 Research and Development Project, Doc. No.:DOH91-NH-1040)
} 
data of the Organization for Economic Co-operation and Development (OECD) countries, the proportion of health care costs as a percentage of Gross Domestic Product (GDP) continues to rise in these years. For example, from 1970 to 2001, health care costs rose from $6.9 \%$ to $13.9 \%$ in the United States' (US) GDP, $5.6 \%$ to $10.9 \%$ of Switzerland's, and $4.0 \%$ to $9.0 \%$ of Belgium's. In Taiwan, health care accounted for $3.4 \%$ of GDP in 1980 and $5.9 \%$ in 2003. Another impetus for payment standards reform is to better allocate health care resources, as the payment system can create economic incentives to influence medical professionals to chose specialties that are needed as well as practice locations in need (Hsiao et al. 1988a, 1988b).

Taiwan's national health insurance (NHI) system initiated in 1995 is a single-insurance provider system. The Bureau of National Health Insurance (BNHI) is the sole institution providing payment to health care providers. One of the most obvious impacts of implementing the NHI system is the changing distribution of physicians among various specialties. Economic incentives related to payment standards play a very important role in this change. Regardless of which type of payment system is used by a health insurance provider, the definition of payment standards within the system is one of the critical factors influencing the quality and cost of health care overall.

There have been numerous studies to determine health care payment standards, according to research findings by Hsiao, Braun and Dunn, et al (1988a), that touch upon many tangible cost considerations such as time of doctor visits, medical equipment, real estate depreciation, employee wages, and so on. Also, there are many intangible costs such as the mental and physical pressures, the amount of effort involved in different types of patient consultations, differences in specialty training requirements, etc (Hsiao et al. 1988a, 1988b, Jegers et at. 2002). Therefore, the BNHI invited many experts' from different specialties to give their opinions, and used those opinions as a basis for discussing setting or adjusting payment standards. Finding and establishing an impartial, fair and reasonable means of defining payment standards is a topic worthy of research.

From the literature review, commonly used methods include the in-depth interview method, the focus group method, the nominal group method and the Delphi method (Chapple and Rogers, 1998; Hoddinott and Pill, 1997; Giacomini and Cook, 2000; Malterud, 2001; Pope and Mays, 1995; Jones, 1995; Gupta and Clarke, 1996). However, these methods are limited by the innate prejudices of specialists' opinions and the fact that they are mainly asked to return qualitative data. It is therefore difficult to convert the opinions of these specialists into objective quantitative values, or to set payment standards based on their conclusions.

The analytic hierarchy process (AHP) developed by Saaty at the University of Pittsburgh in 1971 is applied in this research (Saaty, 1977 and Saaty, 1980). This is a process based on qualitative data, that is based on a mathematical approach that can be used to convert qualitative data from experts' opinions into quantitative data. Results obtained through this method have the advantages of traditional qualitative analysis as well as those of quantitative data. 
The purpose of this study is to constructing a model to obtain consensus from surgical professionals about the importance of "factors related to establishing payment standards in the NHI program." Our model was constructed mainly using the AHP method. The reference factors or criteria, as defined in the Hsiao's "resource-based relative value scale" (RBRVS) were used as a blueprint in setting up the AHP model. The factors in Hsiao's RBRVS were then modified after performing an opinion survey by the Delphi method among surgical specialists in Taiwan and used to construct an AHP model. Finally, an empirical study was carried out to combine the opinions of the experts about the importance of the various factors.

\section{Methods}

\subsection{Subjects}

Before beginning the study, we first explained the research methods and goals to the directors of nine surgical sub-specialty associations. After receiving their approval, nine associations were requested by email or telephone to select from 2 to 9 physicians to represent their sub-specialty according to the number of association members. The nine associations are: general surgery, orthopedic surgery, neurosurgery, cardio-vascular surgery, thoracic surgery, plastic-reconstructive surgery, proctology, urology, and pediatric surgery. These representatives formed the surgery association's NHI working group and served as a consultation group for this study. There were 26 physicians in the NHI working group.

\subsection{Delphi Method}

The Delphi questionnaire was designed based on the RBRVS system with added elements from the results of our literature review. Two rounds of surveys were conducted with each specialization group for the purpose of modifying the factors in RBRVS and determining the importance of these factors in establishing payment standards in the NHI program.

\subsection{Establishing the AHP Model}

The AHP model for the surgical specialty (see Fig. 1) was constructed based on the responses from the surgical specialists through the Delphi method. With the goal of building consensus on "factors related to establishing payment standards in the NHI program," the first tier contains four evaluation aspects: physician's total work input, physician's practice costs, physician's malpractice costs and specialty training costs. The second tier evaluation criteria include 16 items in four groups: time required to perform the service, mental and decision-making effort invested, technical skill and physical effort, mental effort and judgment, psychological stress, pre-service and post-service work, personnel wages, medical supplies, medical equipment, office rents, iatrogenic errors, medical disputes, patient or family violence, risk of injury (from medical instruments) or infection (from serious contagious disease), basic specialty techniques, difficult specialty techniques, and rare specialty techniques.

\subsection{Empirical Study of AHP Model}

On March 15, 2003, the surgical association's NHI working group meeting was held with 26 participants from surgical specialties. 
Goal

Aspects
Criteria

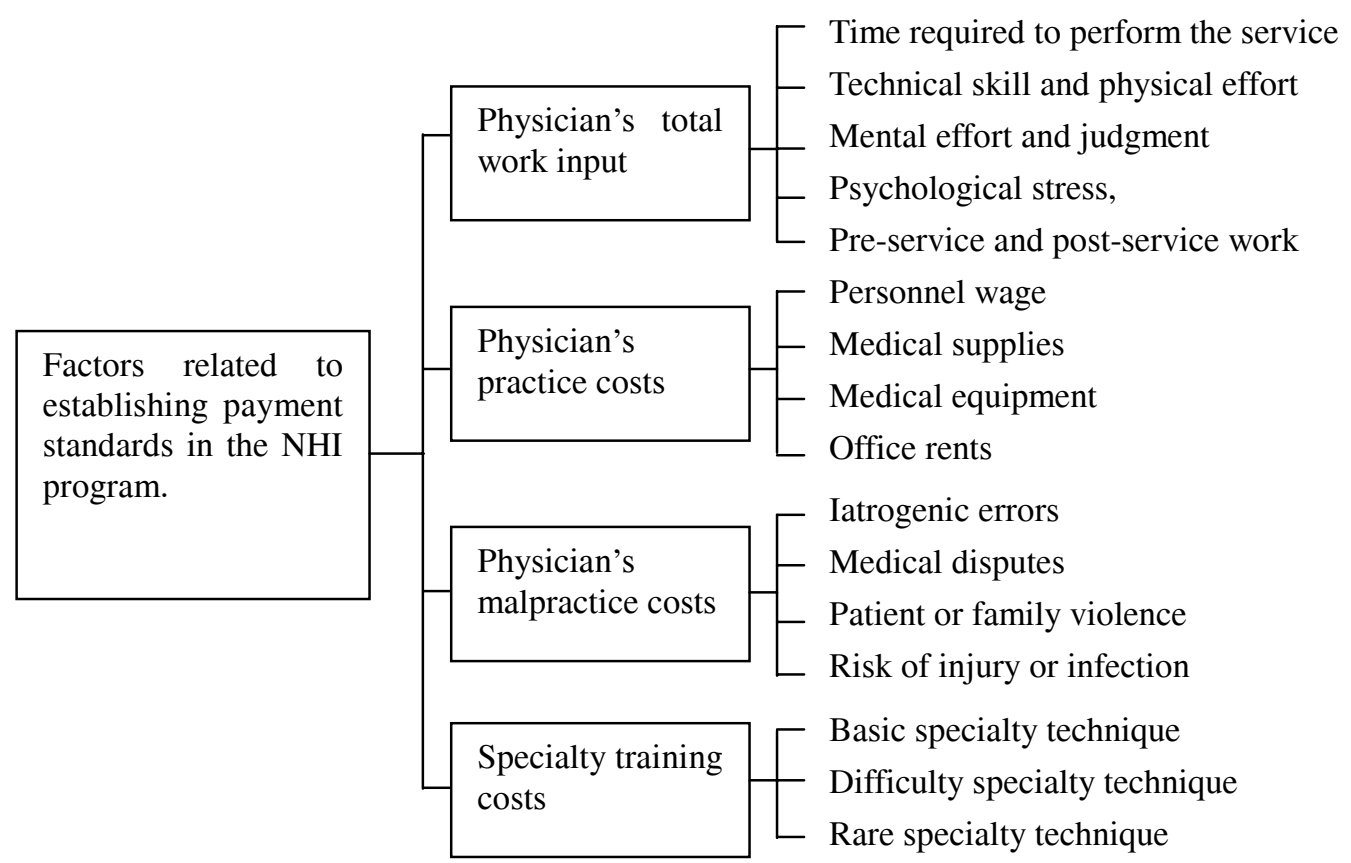

Figure1 The AHP model constructing for the surgical specialty

Representatives of each sub-specialty were invited to participate in the study and respond to the AHP model survey. During the process of the empirical study, every effort was made to provide all specialists access to the same information, and to conduct the surveys in the same way for each participant so that the results would return the best possible relative values for payment standards.

The reference materials provided to each participant included database analysis results from Taiwan's National Health Research Institute from the years 1997 to 2001, proportions of medical costs of each medical specialty, an analysis of personnel figures for each specialty, literature review results and related reference data from foreign countries. The relative value concept was explained in the meeting, followed by an explanation of the factors in the AHP model. After ensuring that each respondent fully understood how to complete the survey questionnaire, the surveys were given to respondents to complete.

After they were completed, the results were compiled using the AHP software product Expert Choice Pro (version 9.5) to compute priority values, consistency indices, consistency ratios and relative weighting valuations for each participant; and Microsoft Excel (version XP) was used to combine their results to give the group's overall relative weighting of the factors. 
Table 1 Three character-distributions of 26 participants

\begin{tabular}{lclccc}
\hline $\begin{array}{c}\text { Surgical } \\
\text { sub-specialties }\end{array}$ & Participants & Service hospital level & Participants & Seniority (years) & Participants \\
\hline General & 9 & Medical center & 12 & Up to 10 & 5 \\
Orthopedic & 3 & Regional hospital & 8 & $11 \sim 20$ & 13 \\
Plastic & 2 & Local hospital & 4 & $21 \sim 30$ & 6 \\
Neurology & 2 & Basic Clinic & 2 & More than 31 & 2 \\
Proctology & 2 & & & & \\
Urology & 2 & & & & \\
Pediatric & 2 & & & & \\
Cardiovascular & 2 & & & & \\
Thoracic & 2 & & 26 & & \\
Total & 26 & & & & \\
\hline
\end{tabular}

Table2 The relative weightings in valid 25 survey responses

\begin{tabular}{lcc}
\hline Aspects and Criteria & First-tier weighting & Second-tier weighting \\
\hline Physician's total work input & $\mathbf{0 . 3 9 3 ( 1 )}$ & 0.065 \\
Time required to perform the service & $\mathbf{0 . 0 9 1 ( 2 )}$ \\
Technical skill and physical effort & $\mathbf{0 . 0 9 3 ( 1 )}$ \\
Mental effort and judgment & $\mathbf{0 . 0 8 3 ( 4 )}$ \\
Psychological stress, & 0.061 \\
Pre-service and post-service work & $\mathbf{0 . 1 6 2 ( 4 )}$ & \\
Physician's practice costs & & $\mathbf{0 . 0 8 0 ( 5 )}$ \\
Personnel wage & 0.031 \\
Medical supplies & & 0.035 \\
Medical equipments & & 0.016 \\
Office rents & & \\
Physician's malpractice costs & $\mathbf{0 . 2 5 1 ( 2 )}$ & 0.055 \\
Iatrogenic errors & & 0.067 \\
Medical disputes & & 0.059 \\
Patient or family violence & & 0.062 \\
Risk of injury or infection & & 0.051 \\
Specialty training costs & & $\mathbf{0 . 0 8 9 ( 3 )}$ \\
Basic specialty technique & & 0.054 \\
Difficulty specialty technique & & \\
Rare specialty technique & & \\
\hline
\end{tabular}




\section{Results}

\subsection{Characters of Participants}

$100 \%$ of the surveys were returned by surgery respondents $(26 / 26)$, and $96 \%$ of those returned were valid (25/26). One survey was invalid as it did not pass the AHP consistency verification tests.

The 26 physicians participating in the study were divided to three groups according to the characters of surgical sub-specialties, service hospital levels and years of seniority (see Table 1). Of these, general surgery accounted for $34.6 \%$ (9/26), the largest proportion. In terms of service hospital level, medical centers accounted for the highest percentage in surgery respondents, with $46.2 \%$ (12/26) employed at medical centers. As for seniority, the majority of subjects in surgery had between 11 and 30 years of seniority, $73.1 \%$ (19/26), respectively. The highest level of seniority in surgery was 39 years (neurosurgery).

\subsection{Rank and Relative Weights of AHP Valuation Factors}

See Table 2 for the analysis of relative weightings in valid survey responses. In the first tier evaluation aspects, the rankings of factors among the 25 surgery respondents were: (1) physician's total work input (0.393); (2) physician's malpractice costs (0.251); (3) specialty training costs (0.194); and (4) physician's practice costs $(0.162)$. Of the 16 second tier factors, the top five ranked as follows: (1) mental effort and judgment (0.093); (2) technical skill and physical effort (0.091); (3) difficult specialty techniques (0.089); (4) psychological stress (0.083); and (5) personnel wages $(0.080)$.

\section{Discussion}

The RBRVS system has been used in the US Medicare system for more than ten years and has been accepted by a majority of physicians as a reasonable payment standard (Harris-Shapiro, 1998, Rotarius, 2001 and Schackleford, 1999). Grimaldi's review of the results concluded that physician workloads accounted for $50 \%$ of Medicare payments, while practice costs accounted for $46 \%$, and malpractice insurance accounted for 4\% (Grimaldi, 2002).

As shown in Table 2, this study found that for the first tier factors, the surgery specialists' judged the physician's total work input to be the most important, at $39.3 \%$. This is not far from the results in the US after implementing the RBRVS system. The second ranked factor was physicians' malpractice costs, at $25.1 \%$. This is very different from that of the RBRVS results in the US and from it one can deduce that Taiwanese surgeons are far more concerned with issues such as medical disputes arising from their practice or violence from dissatisfied patients than are their American counterparts.

The third ranked factor was "specialty training costs". At $19.4 \%$, it was close to practice operating costs. Practice operating costs among Taiwanese physicians accounted for only $16.2 \%$ of the total, whereas in the US, that figure was $46 \%$. A possible reason for this is that the American medical system is an open system, wherein doctors can choose multiple locations in which to conduct their practice, and their operating costs are comparatively high. In Taiwan, the medical system is a closed system in which most hospitals limit physicians to 
working in a single location. Because most physicians work in a single hospital, they are less affected by practice costs and therefore less concerned, or recognize this factor less in setting payment standards.

The key feature of the AHP method is to use the factors (criteria) related to policy decisions to create a hierarchic structure, and then conduct comparative analyses of significance according to policymakers' subjective perceptions. The results in this study indicate that an AHP model designed for surgical specialists is easy for the practicing physicians to understand and to implement. Moreover, it was possible to effectively achieve consensus among numerous specialists and policymakers. Using the AHP, the complex evaluation factors for relative value can be displayed in a simple hierarchical structure that includes all the relevant variables. By using this method, we can display many complex evaluation factors for relative values in medical payment standards in a simple hierarchical structure. After experts in the field perform comparative evaluations, these factors can be ranked according and associated with specific numerical values.

The relative weights of the factors in this study represent the results of consensus reached among different surgical sub-specialty groups. The results can be directly used to formulate standard values as the basis for payment standards. This study focused only on surgical specialists. We recommend that in future studies, the same method and model can be used with other specialties. Studies can then be expanded to cover multiple fields and the results can be used as reference values in cross-specialty situations. Because the final values are meaninful ratio numbers this type of model can be used as a basis for future payment system reforms. Once relative values for a particular specialty have been found, using the above research method they can be expanded to form the basis for setting payment standards for inter-specialty payments.

In summary, the analytic hierarchy process (AHP) as defined by Saaty includes an exacting calculation procedure so that conclusions will have the advantages of both qualitative data and quantitative data, thereby overcoming some of the traditional limitations of qualitative data research, such as biased opinions among specialists and difficulties in finding consensus. The AHP system offers an effective means to gain consensus among different specialty groups, and is extremely useful in integrating the opinions of different groups and resolving complex, multiple-factor policymaking issues. Additionally, the consensus-derived ranking values arrived at through the AHP calculation process are persuasive and are therefore well suited for defining payment standards.

\section{References}

[1] Chapple, A. \& Rogers, A. (1998). Explicit guidelines for qualitative research: a step in the right direction, a defence of the soft option, or a form of sociological imperialism? Family Practice, 15: 556-561

[2] Giacomini, M.K. \& Cook, D.J. (2000). Users' guides to the medical literature, XXIII: qualitative research in health care A, are the results of the study valid? Journal of the American Medical Association, 284: 357-362

[3] Grimaldi, P.L. (2002). Medicare fees for 
physician services are resource-based. Journal of Health Care Finance, 28: 88-104

[4] Gupta, U.G. \& Clarke, R.E. (1996). Theory and applications of the Delphi technique: a bibliography (1975-1994). Technological Forecasting and Social Change, 53: 185-211

[5] Harris-Shapiro, J. (1998). RBRVS revisited. Journal of Health Care Finance, 25: 49-54

[6] Hoddinott, P. \& Pill, R. (1997). A review of recently published qualitative research in general practice: more methodological questions than answers? Family Practice, 14: 313-319

[7] Hsiao, W.C., Braun, P., Dunn, D., et al. (1988a). Resource-based relative values, an overview. JAMA, 260: 2347-2352

[8] Hsiao, W.C., Braun, P., Dunn, D., et al. (1988b). Special report: results and policy implications of the resource-based value study. New England Journal of Medicine, 319: $881-888$

[9] Jegers, M., Kesteloot, K., Graeve, D.D., et al. (2002). A typology for provider payment systems in health care. Health Policy, 60: 255-273

[10]Jones, R. (1995). Why do qualitative research ? British Medical Journal, 311: 2, 1995.

[11]Malterud, Q.K. (2001). Qualitative research: standards, challenges, and guidelines. The Lancet, 358: 483-488

[12]Pope, C. \& Mays, N. (1995). Qualitative research: reaching the parts other methods cannot reach: an introduction to qualitative methods in health and health care services research. British Medical Journal, 311: $42-45$

[13]Rotarius, T. (2001). An RBRVS approach to financial analysis in health care organizations. The Health Care Manager, 19: 17-23

[14]Saaty, T. L. (1977). A scaling method for priorities in hierarchical structures. Journal of Mathematical Psychology, 15: 234-281

[15]Saaty, T. L. (1980). The Analytic Hierarchy Process, New York, McGraw-Hill, 1980

[16]Schackleford, J.L. (1999). Measuring productivity using RBRVS cost accounting. Health Care Financial Management, 53: 67-69

Yuan-Huei Huang is a Visiting Staff of Department of Surgery, Hsin-Chu General Hospital. He received his bachelor's degree in 1987 from the Department of Medicine, College of Medicine at the Chung-Shan Medical University, Taiwan, China. He has also been studying at the Ph.D. Program of Institute of Technology Management, National Chiao Tung University, Taiwan, China, since 1999. His main research interests are health economics, analytic hierarchy process, fuzzy set theory, multiple criteria decision making, and technology management.

Pei-Yeh Chang is the Chief of Department of Pediatric Surgery, Chang Gung Children's Hospital, Taiwan, China. He received his bachelor's degree in 1978 from the Department of Medicine, College of Medicine at the National Taiwan University, China. He has been also serving as an Associate Professor of School of Medicine, National Cheng Kung University, Taiwan, China at present. Besides medical professional, his main research interests are health policy making, medical resource and 
workforce allocation, and medical education.

Chih-Young Hung serves as the Director and an associate professor of Institute of Technology Management in the Faculty of Management, National Chiao Tung University where he has taught since 1991. His $\mathrm{PhD}$ is from the Texas Tech University, U.S.A. He received his bachelor's degree in 1979 from the Department of Electrical and Control Engineering at the National Chiao Tung University, Taiwan, China. His background provided him a special emphasis on the high technology industries in Taiwan, China. He has published a series of papers in studying industry evolution from finance perspective, and also several professional books on financial related areas.

Kuei-Ing Wang serves as the lecturer of Department of Information Management, Ming Hsin University of Science and Technology where she has taught since 1998. She received her MBA degree in 1998 from the Department of Business Administration at the National Chengchi University, Taiwan, China. She has also been studying at the Ph.D. Program of Institute of Technology Management, National Chiao Tung University since 2001. Her main research interests are health economics, the analytic hierarchy process, hi-tech industry analysis, multiple criteria decision making, and information management.

King-Jen Chang is the President of the Foundation of Breast Cancer Prevention and Treatment in Taiwan, China. He has been also serving as a professor of School of Medicine at National Taiwan University, Taiwan, China, since 1991. His $\mathrm{PhD}$ is from the College of Medicine at the National Taiwan University, Taiwan, China, in 1982. Besides medical professional, his main research interests are health economics, health policy making, and medical resource allocation. 